\title{
A GENERALIZED EIGENFUNCTION EXPANSION OF THE GREEN'S FUNCTION
}

\section{MAURICE MACHOVER}

We consider the problem

$$
(L-\lambda) x=0, \quad U x=0,
$$

where $L$ is the ordinary differential operator given by $L x(t)=p_{0}(t) x^{(n)}+p_{1}(t) x^{(n-1)}+\cdots+p_{n}(t) x, \quad p_{0}(t) \neq 0$ on $[a, b]$, and $U$ is the vector boundary form given by

$$
U_{i} x(t)=\sum_{j=1}^{n}\left(a_{i j} x^{(j-1)}(a)+b_{i j} x^{(j-1)}(b)\right), \quad i=1, \cdots, n .
$$

Here the $p_{j}(t)$ are complex-valued functions in $C^{n-j}[a, b]$, and the $a_{i f}$ and $b_{i j}$ are complex. It is no restriction to take $p_{0}(t) \equiv 1$. Corresponding to (1) we have the adjoint problem

$$
\left(L^{+}-\bar{\lambda}\right) y=0, \quad U^{+} y=0,
$$

where $L^{+}$and $U^{+}$are determined by the usual requirement that, for all $x(t), y(t) \in C^{n}[a, b]$ such that $U x=0, U^{+} y=0$,

$$
\int_{a}^{b} \bar{y} L x d t=\int_{a}^{b} x\left[L^{+} y\right]^{-} d t .
$$

Let $G(t, \tau, \lambda)$ be the Green's function for (1). Then, if (1) is selfadjoint, we have the well-known expansion

$$
G(t, \tau, \lambda)=\sum_{n=1}^{\infty} \frac{x_{n}(t) \bar{x}_{n}(\tau)}{\lambda_{n}-\lambda}
$$

where $\lambda_{n}$ and $x_{n}$ are the eigenvalues and eigenfunctions of (1), respectively. In this paper we generalize the expansion to the nonselfadjoint case. In our expansion we will make use of generalized eigenfunctions. A generalized eigenfunction of rank $r$ for (1) is any solution of

$(L-\lambda)^{r} x=0,(L-\lambda)^{r-1} x \neq 0, U x=0, U(L-\lambda) x=0, \cdots, U(L-\lambda)^{r-1} x=0$.

If $x$ is of rank $r$, then $x,(L-\lambda) x, \cdots,(L-\lambda)^{r-1} x$ form a chain of

Received by the editors December 11, 1963. 
generalized eigenfunctions of decreasing rank. Thus if $\lambda_{1}, \lambda_{2}, \cdots$ are the eigenvalues of (1) ordered, say, by increasing modulus, the space of generalized eigenfunctions for $\lambda_{j}$ of rank less than or equal to $m_{j}$ is the solution space of

(3) $\left(L-\lambda_{j}\right)^{m_{j} x}=0, U x=0, U\left(L-\lambda_{j}\right) x=0, \cdots, U\left(L-\lambda_{j}\right)^{m_{j}-1} x=0$.

There exists a basis of (3) made up of chains of generalized eigenfunctions, and all such chain bases have the same number and lengths of chains [1]. We denote such a chain basis by

(4) $\quad x_{k l}^{(j)}(t), \quad j=1,2, \cdots ; k=1,2, \cdots, c_{j} ; l=1,2, \cdots, r_{j k}$.

Here the first subscript denotes chain number, the second denotes rank, $c_{j}$ is the number of chains corresponding to $\lambda_{j}, r_{j k}$ is the length of the $k$ th chain for $\lambda_{j}$. We assume the chains so ordered that $m_{j} \geqq r_{j 1}$

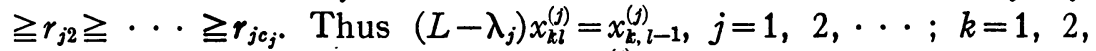
$\cdots, c_{j} ; l=1,2, \cdots, r_{j k}$. (We take $\left.x_{k 0}^{(j)} \equiv 0\right)$.

Corresponding to (3) we have the adjoint problem

(5) $\left(L^{+}-\bar{\lambda}_{j}\right)^{m_{j}} \psi=0, U^{+} \psi=0, U^{+}\left(L^{+}-\bar{\lambda}_{j}\right) \psi=0, \cdots, U^{+}\left(L^{+}-\bar{\lambda}_{j}\right)^{m_{j}-1} \psi=0$,

with the adjoint basis $\psi_{k l}^{(j)}(t)$. The $\psi$ basis agrees with the $x$ basis in rank, number, and lengths of chains. In particular, there exists the corresponding chain basis to (4) such that

(6) $\quad \int_{a}^{b} x_{m n}^{(j)}(t) \Psi_{m^{\prime} n^{\prime}}^{(j)}(t) d t=\delta_{m m^{\prime}} \delta_{n, r_{j m+1-n^{\prime}}}$ (i.e., the scalar product).

(Such a corresponding chain basis may be obtained by starting with any $\psi$ basis and using (6) to obtain the appropriate linear combinations.)

Our generalized expansion (indeed, the very fact that an infinite sequence of eigenvalues exist) will hold for what G. D. Birkhoff [2] has called regular boundary conditions. To define regularity we first normalize the boundary form $U$ as follows:

Reduce the number of conditions $U_{i} x=0$ of order $n-1$ (that is, containing either $x^{(n-1)}(a)$ or $\left.x^{(n-1)}(b)\right)$ to a minimum, at most two, by linear combination. Then, in the remaining conditions, reduce those of order $n-2$ to a minimum, at most two, again by linear combination. Continue in this way as long as conditions remain. The normalized conditions will have the form

$$
U_{i} x=U_{i a} x+U_{i b} x=0, \quad i=1, \cdots, n,
$$

where 


$$
\begin{array}{r}
U_{i a} x=\alpha_{i} x^{\left(k_{i}\right)}(a)+\sum_{j=0}^{k_{i}-1} \alpha_{i j} x^{(j)}(a), \quad U_{i b} x=\beta_{i} x^{\left(k_{i}\right)}(b)+\sum_{j=0}^{k_{i}-1} \beta_{i j} x^{(j)}(b), \\
n-1 \geqq k_{1} \geqq k_{2} \geqq \cdots \geqq k_{n},
\end{array}
$$

where no three consecutive $k$ 's are the same.

Definition. Let $\omega_{i}, i=1, \cdots, n$, be the $n$ roots of unity. Let the $\omega_{i}$ be taken in every order such that, for some $\rho \neq 0$,

$\operatorname{Re}\left(\rho \omega_{1}\right)<\operatorname{Re}\left(\rho \omega_{2}\right)<\cdots<\operatorname{Re}\left(\rho \omega_{n}\right) \quad(\operatorname{Re} x$ denotes the real part of $x$ ).

Then, if $n=2 \mu-1$ is odd and always neither $\theta_{0}=0$ nor $\theta_{1}=0$, where $\theta_{0}$ and $\theta_{1}$ are defined by the identity

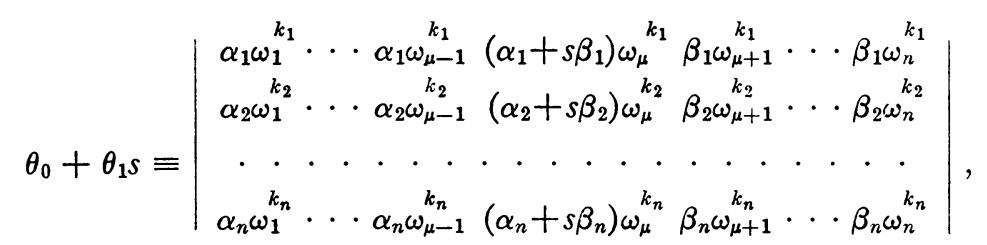

or if $n=.2 \mu$ is even and always neither $\theta_{1}=0$ nor $\theta_{2}=0$, where $\theta_{0}, \theta_{1}$, and $\theta_{2}$ are defined by the identity

$$
\begin{aligned}
& \theta_{0}+\theta_{1} s+\frac{\theta_{2}}{s}
\end{aligned}
$$

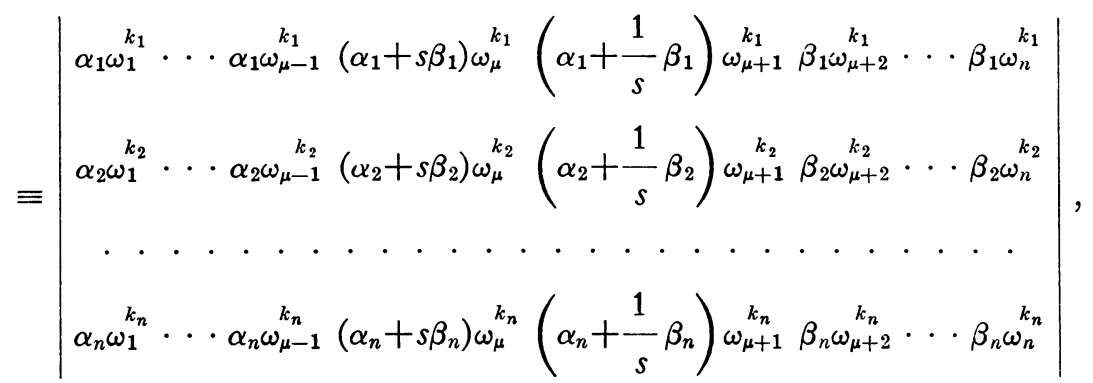

the conditions $U x=0$ are said to be regular. For $n=2$ the standard Sturm-Liouville conditions $h x(a)+k x^{\prime}(a)=0, l x(b)+m x^{\prime}(b)=0$, where $m k \neq 0$, are regular. The conditions $x(a)=0, x^{\prime}(a)=k x(b)$ are nonregular.

Our result is summarized in the following theorem.

Theorem. Let (1) be regular with the Green's function $G(t, \tau, \lambda)$. Let $\lambda_{1}, \lambda_{2}, \cdots$ be the eigenvalues of (1) ordered by increasing modulus, with $m_{j}$ the order of $\lambda_{j}$ as a pole of $G$. Let $x_{k l}^{(j)}(t)$ be a chain basis of (3). Let $\psi_{\mathbf{k} l}^{(j)}(t)$ be the corresponding chain basis of (5). Then 


$$
G(t, \tau, \lambda)=\sum_{=1}^{\infty} \sum_{k=1}^{c_{j}} \sum_{l=1}^{r_{j k}} x_{k l}^{(j)}(t)\left[\frac{\bar{\psi}_{k l^{\prime}}^{(j)}(\tau)}{\lambda_{j}-\lambda}-\frac{\bar{\psi}_{k, l^{\prime}-1}^{(j)}(\tau)}{\left(\lambda_{j}-\lambda\right)^{2}}+\cdots\right.
$$

where $l^{\prime}=r_{j k}+1-l$.

$$
\left.+(-1)^{l^{\prime}-1} \frac{\bar{\psi}_{k 1}^{(j)}(\tau)}{\left(\lambda_{j}-\lambda\right)^{l^{\prime}}}\right], \quad t, \tau \in(a, b),
$$

REMARK. When (1) is selfadjoint, all the $m_{j}=1, \psi_{k 1}^{(j)}=x_{k 1}^{(j)}$, and the expansion reduces to (2).

Proof. We make use of two theorems. The first is due to G. D. Birkhoff.

Theorem (BIRKhoff [2]). Let (1) be regular. If $f(t)$ is piecewise continuous with piecewise continuous derivative on $[a, b]$, then

$$
\begin{array}{r}
\frac{1}{2}[f(t+0)+f(t-0)] \\
=\lim _{n \rightarrow \infty}-\frac{1}{2 \pi i} \int_{a}^{b} d t^{\prime} f\left(t^{\prime}\right) \oint_{C_{n}} G\left(t, t^{\prime}, \lambda\right) d \lambda \text { on }(a, b) .
\end{array}
$$

At $t=a$ and $t=b$ the sequence converges to linear combinations of $f(a+0)$ and $f(b-0)$, the coefficients being independent of $f$. Here $C_{n}$ is a circle about the origin of radius $n$ in the $\lambda$-plane.

The second theorem concerns the residue of $G$.

Theorem [1]. Let $G\left(t, t^{\prime}, \lambda\right)$ be the Green's function of (1). If $\lambda_{j}$ is a pole of $G$ of order $m_{j}$, and $x_{k l}^{(j)}(t)$ constitute any chain basis of (3), then the residue of $G$ at $\lambda_{j}$ is

$$
-\sum_{k=1}^{c_{j}} \sum_{l=1}^{r_{j k}} x_{k l}^{(j)}(t) \bar{\psi}_{k l^{\prime}}^{(j)}\left(t^{\prime}\right), \quad l^{\prime}=r_{j k}+1-l,
$$

where $\psi_{\mathbf{k} l}^{(j)}(t)$ is the corresponding chain basis of (5).

Taking $f(t)$ in (8) equal to $G(t, \tau, \lambda)$ and using (9) we have

$$
\begin{aligned}
G(t, \tau, \lambda) & =\lim _{n \rightarrow \infty}-\frac{1}{2 \pi i} \int_{a}^{b} d t^{\prime} G\left(t^{\prime}, \tau, \lambda\right) \oint_{C_{n}} G\left(t, t^{\prime}, \lambda\right) d \lambda \\
& =\sum_{j=1}^{\infty} \sum_{k=1}^{c_{j}} \sum_{l=1}^{r_{j k}} x_{k l}^{(j)}(t) \int_{a}^{b} G\left(t^{\prime}, \tau, \lambda\right) \bar{\psi}_{k l^{\prime}}^{(j)}\left(t^{\prime}\right) d t^{\prime}, \quad t, \tau \in(a, b) .
\end{aligned}
$$

We now evaluate the integral in (10). Since $G\left(t^{\prime}, \tau, \lambda\right)=\bar{G}^{+}\left(\tau, t^{\prime}, \bar{\lambda}\right)$, where $G^{+}$is the Green's function of the adjoint to problem (1) [3],

$$
\int_{a}^{b} G\left(t^{\prime}, \tau, \lambda\right) \bar{\Psi}_{k l^{\prime}}^{(j)}\left(t^{\prime}\right) d t^{\prime}=\left[\int_{a}^{b} G^{+}\left(\tau, t^{\prime}, \bar{\lambda}\right) \psi_{k l^{\prime}}^{(j)}\left(t^{\prime}\right) d t^{\prime}\right]^{-}
$$


If we define $\psi_{\mathbf{k} 0}^{(j)} \equiv 0$, and if $\lambda$ is not an eigenvalue of (1),

$$
\begin{aligned}
\left(L^{+}-\bar{\lambda}\right) \psi_{k l^{\prime}}^{(j)}(t) & =\left(L^{+}-\bar{\lambda}_{j}\right) \psi_{k l^{\prime}}^{(j)}(t)+\left(\bar{\lambda}_{j}-\bar{\lambda}\right) \psi_{k l^{\prime}}^{(j)}(t) \\
& =\psi_{k, l^{\prime}-1}^{(j)}(t)+\left(\bar{\lambda}_{j}-\bar{\lambda}\right) \psi_{k l^{\prime}}^{(j)}(t) .
\end{aligned}
$$

Thus

$$
\psi_{k l^{\prime}}^{(j)}(t)=\int_{a}^{b} G^{+}\left(t, t^{\prime}, \bar{\lambda}\right) \psi_{k, l^{\prime}-1}^{(j)}\left(t^{\prime}\right) d t^{\prime}+\left(\bar{\lambda}_{j}-\bar{\lambda}\right) \int_{a}^{b} G^{+}\left(t, t^{\prime}, \bar{\lambda}\right) \psi_{k l^{\prime}}^{(j)}\left(t^{\prime}\right) d t^{\prime}
$$

Thus

$$
\begin{aligned}
& \int_{a}^{b} G^{+}\left(t, t^{\prime}, \bar{\lambda}\right) \psi_{k l^{\prime}}^{(j)}\left(t^{\prime}\right) d t^{\prime} \\
& \quad=\frac{\psi_{k l^{\prime}}^{(j)}(t)}{\bar{\lambda}_{j}-\bar{\lambda}}-\frac{1}{\bar{\lambda}_{j}-\bar{\lambda}} \int_{a}^{b} G^{+}\left(t, t^{\prime}, \bar{\lambda}\right) \psi_{k, l^{\prime}-1}^{(j)}\left(t^{\prime}\right) d t^{\prime} \\
& \quad=\frac{\psi_{k l^{\prime}}(t)}{\bar{\lambda}_{j}-\bar{\lambda}}-\frac{\psi_{k, l^{\prime}-1}(t)}{\left(\bar{\lambda}_{j}-\bar{\lambda}\right)^{2}}+\frac{1}{\left(\bar{\lambda}_{j}-\bar{\lambda}\right)^{2}} \int_{a}^{b} G^{+}\left(t, t^{\prime}, \bar{\lambda}\right) \psi_{k, l^{\prime}-2}^{(j)}\left(t^{\prime}\right) d t^{\prime} \\
& =\frac{\psi_{k l^{\prime}}^{(j)}(t)}{\bar{\lambda}_{j}-\bar{\lambda}}-\frac{\psi_{k, l^{\prime}-1}(t)}{\left(\bar{\lambda}_{j}-\bar{\lambda}\right)^{2}}+\cdots+(-1)^{l^{\prime}-1} \frac{\psi_{k 1}^{(j)}(t)}{\left(\bar{\lambda}_{j}-\bar{\lambda}\right)^{l^{\prime}}} \cdot
\end{aligned}
$$

From (10), (11), and (12), (7) follows immediately.

This result is clearly anticipated in B. Friedman's Principles and techniques of applied mathematics.

\section{REFERENCES}

1. M. Machover, Generalized eigenvectors and separation of variables, Doctoral Dissertation, New York University, New York, 1963.

2. G. D. Birkhoff, Boundary value and expansion problems of ordinary linear differential equations, Trans. Amer. Math. Soc. 9 (1908), 373-395; also in Collected Mathematical Papers of G. D. Birkhoff, Vol. I, Amer. Math. Soc., Providence, R.I., 1950.

3. E. A. Coddington and N. Levinson, Theory of ordinary differential equations, McGraw-Hill, 1955; Chapters 7, 11, and 12.

FAIRLEIGH Dickinson University 